\title{
Invasión ósea del carcinoma de células escamosas de la cavidad oral, análisis clínico - patológico de 62 casos
}

Bone invasion of squamous cell carcinoma of the oral cavity, clinical-pathological analysis of 62 cases

\author{
Jaqueline Vaz Vanini MSc'; Ana Maria Hoyos Cadavid PhD'; Cláudia Malheiros Coutinho-Camillo PhD²; \\ Leandro Luongo de Matos $\mathbf{M D}^{3}$, Claudio R Cernea $\mathbf{M D}^{4}$, Silvia Vanessa Lourenço $\mathbf{P h D}^{1}$.
}

\begin{abstract}
RESUMEN
Introducción: El pronóstico de pacientes con carcinoma de células escamosas (CEC) de la cavidad oral con presencia de invasión ósea continua sombrío. El patrón de invasión ósea puede ser usado como indicador de agresividad y correlacionado con el comportamiento clínico del tumor. Objetivo: Evaluar los patrones histopatológicos de invasión ósea en pacientes con CEC de la cavidad oral y correlacionarlos con la tasa de sobrevida. Materiales y métodos: La muestra fue constituida por 62 pacientes con presencia de CEC en la cavidad oral e invasión ósea. Las características epidemiológicas e histopatológicas fueron tabuladas y analizadas. Los Test Chi- cuadrado y exacto de Fischer fueron empleados para verificar las asociaciones estadísticas entre los datos. Resultados: Fueron afectados más hombres en la quinta década de vida y observada una fuerte asociación con tabaquismo y etilismo crónico. Fueron relatadas muertes en $58 \%$ de los pacientes, en un periodo de 5 años. El patrón histológico más prevalente fue el infiltrativo, asociado a menores tasas de sobrevida. Conclusión: El análisis del patrón histopatológico de los CEC de la cavidad oral, puede ser usado como factor pronóstico auxiliando en la decisión del abordaje del tratamiento oncológico.
\end{abstract}

PALABRAS CLAVE: Cáncer oral; Carcinoma de células escamosas; Patología (DeCS).

\section{SUMMARY}

Introduction: Prognosis for patients with squamous cell carcinoma (SCC) in oral cavity with bone invasion presence is concerning. Bone invasion patterns can be used to indicate aggressiveness and can be correlated with tumor clinical behavior. Objective: To evaluate bone invasion histopathological patterns in patients with oral cavity SCC and correlate them with the survival rate. Materials and methods: 62 patients with presence of SCC in the oral cavity as well as bone invasion were recruited for this study. Epidemiological and histopathological characteristics were tabulated and analyzed. Fischer's Square and Exact Tests were used to verify any statistical associations between the data. Results: More men were affected, especially in the fifth decade of life, and a strong association with smoking and chronic alcoholism was observed. Deaths were reported in $58 \%$ of patients, over a period of 5 years. The most prevalent histological pattern was the infiltrative, associated with lower survival rates. Conclusion: An analysis of the histopathological patterns of oral cavity SCC can be used as a prognostic factor thereby assisting with the decision of which oncological treatment approach to use.

KEYWORDS: Oral cancer; Squamous cell carcinoma; Pathology (DeCS).

Odontólogas del Departamento de Estomatología, Facultad de Odontología, Universidad de São Paulo, Brasil

Bióloga del Departamento de Patologia, AC Camargo Cáncer Center, São Paulo, Brasil.

Médico del Departamento de Cirugía de Cabeza y Cuello, Facultad de Medicina, Universidad de São Paulo, Brasil.

Médico del Departamento de Cirugía General, Facultad de Medicina, Universidad de São Paulo, Brasil.

Todos los autores contribuyeron de forma significativa en la elaboración y redacción de este trabajo. 


\section{INTRODUCCIÓN}

El carcinoma de células escamosas (CEC) es el más común de los tumores de cabeza y cuello y presenta una tasa de sobrevida de 5 años en aproximadamente $50 \%$ de los pacientes $(1,2)$. Tiene una incidencia mayor en hombres y se encuentra entre las 10 neoplasias más comunes en el mundo (3). La asociación entre tabaquismo y etilismo crónicos son los factores de riesgo más comunes $(3,4)$.

La invasión de estructuras óseas, en general, ocurre en fases más avanzadas de la neoplasia, empeorando el estadio del tumor y exigiendo grandes resecciones quirúrgicas. Este tipo de tratamiento conlleva a defectos estéticos y funcionales, así como a fuertes impactos psicológicos y sociales afectando la calidad de vida $(5,6)$.

A pesar de los avances terapéuticos en los últimos años, muchos pacientes aún tienen una respuesta desfavorable al tratamiento y presentan altas tasas de recidiva (7).

Los tumores que se localizan en la encía, la región retromolar, el piso de boca o el paladar, tienen una mayor proximidad anatómica con el hueso, lo que facilita su diseminación. Cuando el tumor entra en contacto con la estructura ósea, es considerado T4 según la clasificación propuesta por la Organización Mundial de la Salud (OMS) (8). Algunos autores cuestionan esta clasificación basándose en estudios que indican que solo la invasión ósea a nivel medular está relacionada con recidivas y tasas de supervivencia más bajas $(9$, 10,11,12).

El patrón de invasión ósea de los tumores puede ser dividido en tres tipos: erosivo, infiltrativo y mixto (13). El patrón erosivo representa un frente de invasión de tejido óseo bien definido, con presencia de actividad osteoclástica y fibrosis, a diferencia del patrón infiltrativo, caracterizado por conjuntos de células tumorales formando nidos y proyecciones que penetran los sistemas de Havers. Algunos autores relatan un peor pronóstico para el patrón de invasión ósea infiltrativa, sin embargo, los informes de este fenómeno aún son bastante escasos en la literatura $(14,15)$.

Las tasas de mortalidad y morbilidad relacionadas con el tratamiento de pacientes con CEC de la cavi- dad oral son extremadamente altas. Los patrones de invasión ósea, su relación con la evolución de la enfermedad y su asociación con la tasa de supervivencia siguen siendo factores que merecen más atención y un mayor número de estudios, siendo este el principal motivo para la realización de este trabajo.

\section{MATERIALES Y MÉTODOS:}

Fueron seleccionados todos los casos diagnosticados con carcinoma de células escamosas de cavidad oral operados en el servicio de Cirugía de Cabeza y Cuello del Hospital de las Clínicas, de la Facultad de Medicina de la Universidad de São Paulo (HC-FMUSP) entre los años 2006 y 2015, totalizando 493 pacientes. 170 fueron clasificados como T4 y 130 presentaron invasión ósea, según los informes de los exámenes anatomopatológicos de las muestras.

Las láminas de las piezas quirúrgicas de los 130 casos seleccionados, fueron teñidas con hematoxilina-eosina y examinadas en la totalidad de sus cortes por dos observadores previamente calibrados. Fue usado un microscopio óptico (Olympus) sobre un foco fijo y con claridad de campo y se realizó un análisis cualitativo para observar la presencia histológica de invasión ósea, totalizando una muestra de 62 casos después de la aplicación de este criterio.

Los 62 casos fueron clasificados histológicamente según el patrón de invasión ósea predominante (infiltrativo o erosivo), también fue observada la presencia de invasión perineural y angiolinfática y el grado de diferenciación tumoral (bien diferenciado; moderado; poco diferenciado; indiferenciado).

Los datos clínicos y epidemiológicos como sexo, edad, sitio primario y tamaño del tumor, tabaquismo / alcoholismo, metástasis, recidivas y muertes, fueron tabulados y analizados usando los programas Test chi-cuadrado y prueba exacta de Fischer para verificar la presencia de asociación entre ellos. La supervivencia general se comparó entre los grupos mediante la curva de Kaplan-Meier utilizando el programa SPSS ${ }^{\circledR}$ versión 21.0 (SPSS ${ }^{\circledR}$ Inc; Illinois, EE. UU) la probabilidad de error significativo de tipo I se ajustó al $5 \%(\mathrm{p} \leq 0,05)$.

Este estudio fue aprobado por el Comité de ética de la Facultad de Odontología de la Universidad de Sao 
Paulo, bajo el protocolo número 2.201.788, CAAE 42520915.0.0000.0075.

\section{RESULTADOS}

Los casos de neoplasias con invasión ósea confirmada (62 casos) representaron el 12,5\% de todos los casos operados en 9 años en el mismo hospital.

En la muestra estudiada $76 \%$ de los pacientes correspondieron al sexo masculino, en un rango de edad entre 43-91 años y con edad media de 59,4 años. Fueron observadas altas tasas de tabaquismo (65\%) y etilismo crónico (56\%) (Ver tabla 1).

Los tumores presentaron un tamaño promedio de $5,1 \mathrm{~cm}$ en su mayor diámetro, siendo que la mayoría $(77 \%)$ tenía un tamaño igual o mayor que $4 \mathrm{~cm}$. El 58\% de los pacientes fallecieron en un período de tiempo de 5 años, después del tratamiento.

Con relación a la localización del tumor, la región más afectada fue el piso de boca (45\%). El área retromolar represento $(16 \%)$ de los CECs, la lengua (12\%), el paladar duro (8\%), la encía inferior (6\%), la encía superior (5\%), los labios (5\%) y la mucosa yugal (3\%). La recurrencia locorregional estuvo presente en 19 casos $(30,6 \%)$ la mayoría con presencia de invasión perineural $(78,6 \%)$. El patrón histológico de CEC moderadamente diferenciado fue el más común (66\%), seguido de los patrones bien diferenciados (23\%) y poco diferenciados (11\%), como se muestra en la Tabla 1.

Con respecto al patrón de invasión ósea, los casos fueron agrupados según el patrón predominante, siendo que $66 \%$ correspondió al patrón infiltrativo y $34 \%$ al patrón erosivo, ilustrados en la Figura 1.

Fue encontrada asociación estadísticamente significativa entre el patrón histopatológico infiltrativo dominante y el tiempo de supervivencia más corto. Observando una diferencia de 13 meses en la tasa de supervivencia promedio, entre los dos patrones ilustrada en la Figura 2.

Tabla 1 - Características epidemiológicas de los pacientes

\begin{tabular}{lll}
\hline \multicolumn{1}{c}{ Variable } & \multicolumn{1}{c}{ Categoría } & n (\%) \\
\hline \multirow{2}{*}{ Sexo } & Masculino & $47(76 \%)$ \\
Tabaquismo & Femenino & $15(24 \%)$ \\
\multirow{5}{*}{ Etilismo } & Negativo & $22(35 \%)$ \\
& Positivo & $40(65 \%)$ \\
Invasión Perineural & Negativo & $27(44 \%)$ \\
& Positivo & $35(56 \%)$ \\
Invasión Angiolinfática & Negativo & $14(22 \%)$ \\
& Positivo & $48(78 \%)$ \\
Grado de diferenciación & Pegativo & $42(68 \%)$ \\
& Positivo & $20(32 \%)$ \\
& Moderado & $15(23 \%)$ \\
Óbito & Poco diferenciado & $40(66 \%)$ \\
& Si & $36(11 \%)$ \\
& No & $26(48 \%)$ \\
\hline
\end{tabular}




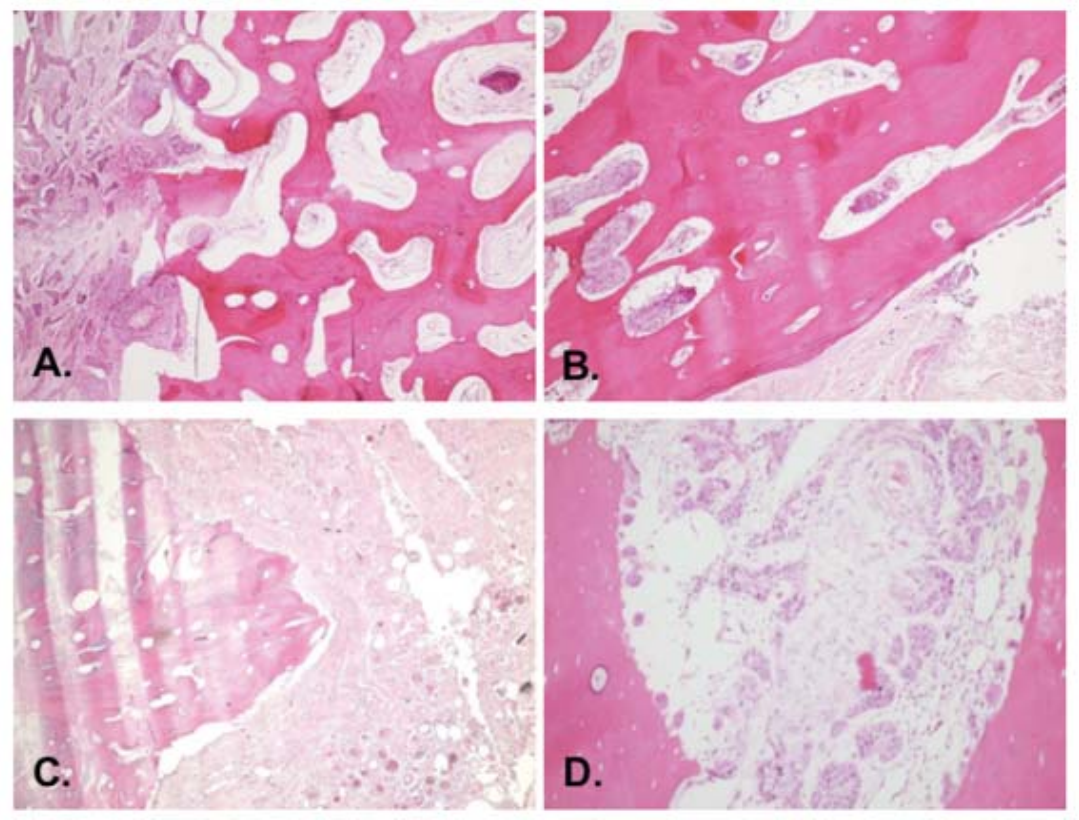

Figura 1: (A-B) CEC con invasión ósea patrón infiltrativo (HE x4) (C) CEC con invasión ósea patrón erosivo ( $\mathrm{HE} \mathrm{x4)} \mathrm{(D)} \mathrm{CEC} \mathrm{con} \mathrm{invasión} \mathrm{ósea} \mathrm{patrón}$ erosivo y actividad osteoclástica (HE x10).

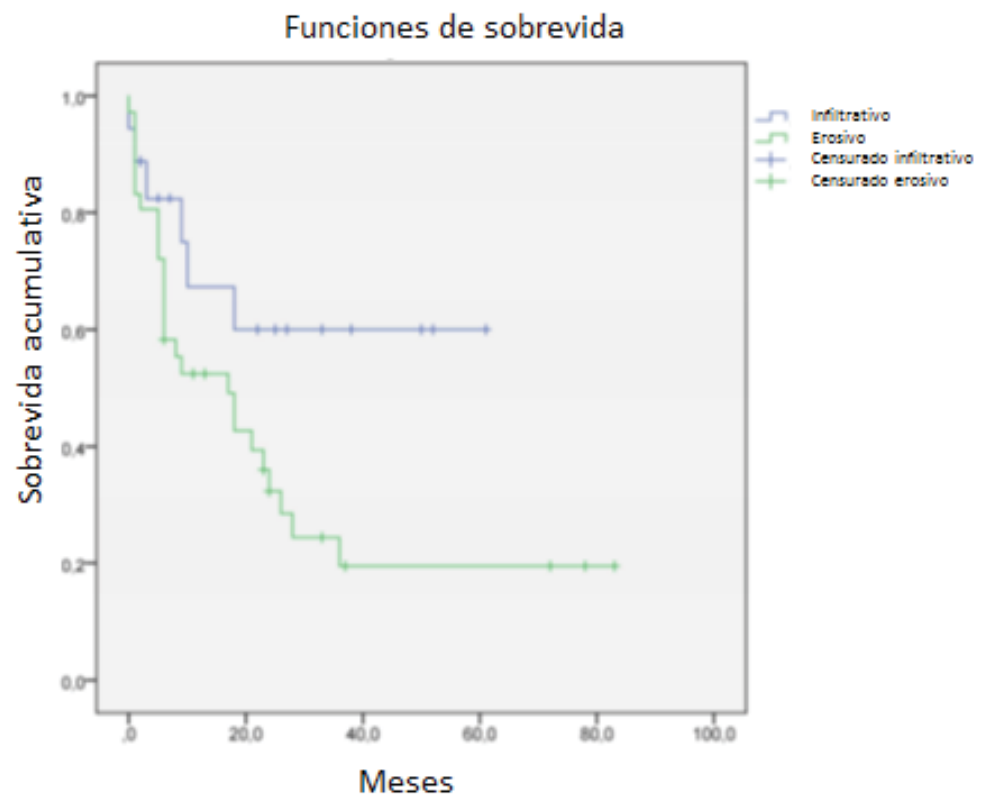

Figura 2: Menor supervivencia general en el patrón predominante infiltrativo (rango logarítmico 0.048, promedio patrón erosivo - verde - 39.64 meses, promedio patrón infiltrativo - azul - 26.11 meses). 
Paulo, bajo el protocolo número 2.201.788, CAAE 42520915.0.0000.0075.

\section{RESULTADOS}

Los casos de neoplasias con invasión ósea confirmada (62 casos) representaron el 12,5\% de todos los casos operados en 9 años en el mismo hospital.

En la muestra estudiada $76 \%$ de los pacientes correspondieron al sexo masculino, en un rango de edad entre 43-91 años y con edad media de 59,4 años. Fueron observadas altas tasas de tabaquismo $(65 \%)$ y etilismo crónico (56\%) (Ver tabla 1).

Los tumores presentaron un tamaño promedio de $5,1 \mathrm{~cm}$ en su mayor diámetro, siendo que la mayoría $(77 \%)$ tenía un tamaño igual o mayor que $4 \mathrm{~cm}$. El 58\% de los pacientes fallecieron en un período de tiempo de 5 años, después del tratamiento.

Con relación a la localización del tumor, la región más afectada fue el piso de boca (45\%). El área retromolar represento $(16 \%)$ de los CECs, la lengua (12\%), el paladar duro (8\%), la encía inferior (6\%), la encía superior (5\%), los labios (5\%) y la mucosa yugal (3\%). La recurrencia locorregional estuvo presente en 19 casos $(30,6 \%)$ la mayoría con presencia de invasión perineural (78,6\%). El patrón histológico de CEC moderadamente diferenciado fue el más común (66\%), seguido de los patrones bien diferenciados (23\%) y poco diferenciados (11\%), como se muestra en la Tabla 1.

Con respecto al patrón de invasión ósea, los casos fueron agrupados según el patrón predominante, siendo que $66 \%$ correspondió al patrón infiltrativo y $34 \%$ al patrón erosivo, ilustrados en la Figura 1.

Fue encontrada asociación estadísticamente significativa entre el patrón histopatológico infiltrativo dominante y el tiempo de supervivencia más corto. Observando una diferencia de 13 meses en la tasa de supervivencia promedio, entre los dos patrones ilustrada en la Figura 2.

\section{DISCUSION}

El carcinoma de células escamosas de la cavidad oral (CEC) representa el 5,6\% de todos los tumores malignos, siendo el sexto más común en el mundo. $\mathrm{Su}$ incidencia anual es de aproximadamente 263,000 casos y la tasa de mortalidad es de aproximadamente 128,000 por año (16). A pesar de los importantes avances diagnósticos y terapéuticos conseguidos en los últimos años, las tasas de supervivencia no han podido mejorarse en las últimas décadas y permanecen por debajo del $50 \%$ (17).

En concordancia con lo relatado en la literatura, nuestro estudio mostró que los hombres son más acometidos por el CEC que las mujeres en una proporción de 3: 1 , siendo más frecuente en la quinta década de la vida y fuertemente asociado con tabaquismo y etilismo crónico $(2,3,15,18)$.

Cuanto a prevalencia de localización, fue difícil comparar este estudio con otros, ya que el nuestro tenía como criterio de inclusión tumores con presencia de invasión ósea, siendo este tipo de estudios escasos en la literatura. El piso de boca fue la región más afectada (45\%), seguida del área retromolar (16\%). En su estudio, Chen YL et al., 2011 (15) encontraron mayor prevalencia en la mucosa oral (44.2\%) seguida del trígono retromolar $(27,9 \%)$.

La proximidad anatómica del piso de boca y el área retromolar con el hueso es la explicación más probable de la incidencia de invasión en estas regiones de la cavidad oral. Es importante resaltar que en este estudio solo se analizaron casos con invasión ósea histológicamente comprobada, lo que explica la diferencia en relación con la incidencia global de la enfermedad, donde la lengua suele ser la estructura más comúnmente afectada por los tumores primarios (17).

La presencia de invasión ósea caracteriza un factor pronóstico menos favorable, incluso en tumores menores que $4 \mathrm{~cm}$ (11). Michalek J et al., 2019 (19) informaron que algunas citocinas como TNF $\alpha$ y PTHrP conducen a la activación del receptor de expresión del ligando NF-kB (RANKL) o a la supresión de la osteoprotegerina (OPG) que estimula la osteoclastogénesis. Las células displásicas producen entonces enzimas que destruyen el tejido óseo y contribuyen a la migración de las células tumorales a los tejidos adyacentes. Además, estas enzimas proteolíticas actúan principalmente sobre los precursores de osteoclastos para promover su diferenciación y maduración $(6,19,20,21)$.

Otros autores como Takayama Y et al., En 2010 (22), Cui N et al., 2010 (23) y más recientemente Michalek 
J et al., 2019 (19) informaron que los tumores con presencia de invasión ósea liberan factores de crecimiento como el factor de crecimiento de fibroblastos (FGF) y el factor de crecimiento transformador- $\boldsymbol{\beta}$ (TGF- $\boldsymbol{\beta}$ ), lo que aumenta la cantidad de citosinas relacionadas con los osteoclastos y provoca la progresión tumoral.

A pesar de los numerosos estudios y el interés constante por parte de los patólogos por la comprensión de la fisiopatología de los CEC, todavía no existe un consenso sobre la interpretación de los criterios histopatológicos y los patrones de invasión ósea.

Namim AW et al, en 2019 (8) realizaron un estudio con 74 casos de CEC con presencia de invasión ósea y a diferencia de nuestro estudio, el patrón predominante fue el erosivo (53\%). Los autores informan que, si bien este patrón está bien descrito en la literatura, a menudo se malinterpreta porque no existe una definición aceptada de invasión mandibular por parte del Colegio de Patólogos Americanos (College of American Pathologist) y en segundo lugar, porque el patrón erosivo exhibe una banda de células estromales que separa el carcinoma de la mandíbula, lo que dificulta la comprensión conceptual de la invasión histológica (8).

Estudios actuales sugieren que la invasión ósea puede comenzar con un patrón erosivo y evolucionar hacia un patrón infiltrativo $(19,24)$. Siguiendo esta hipótesis, los casos mixtos encontrados en nuestro estudio podrían estar en un proceso intermedio de transición de invasión ósea.

El patrón infiltrativo, predomínate en este estudio, tiene un pronóstico menos favorable, confirmado por el análisis de supervivencia global en la muestra estudiada, probablemente causado por la mayor dificultad para lograr márgenes quirúrgicos limpios. Las características microscópicas irregulares de este tipo de invasión histológica aumentan la posibilidad de que los grupos de células tumorales permanezcan en el sitio del tumor primario incluso después del tratamiento, causando recidivas (15).

A medida que los CEC progresan, invaden los tejidos circundantes lo que aumenta la posibilidad de comprometer el hueso. Chen et al., En 2011 (15) informaron en su trabajo una incidencia de invasión ósea del $16,3 \%$, mientras que nuestro estudio mostró un porcentaje ligeramente menor $(12,6 \%)$.
Los datos encontrados en esta muestra sobre el grado de diferenciación tumoral están de acuerdo con los reportados en la literatura, donde el patrón pobremente diferenciado del CEC caracteriza una enfermedad más agresiva (1).

Aunque en nuestro estudio la mayoría de los casos no presentaron metástasis a distancia, un porcentaje significativo tuvo recidiva locorregional $(30,6 \%)$, a diferencia del estudio realizado por Chen YL et al, (15) donde el porcentaje fue menor (8.3\%), lo que demuestra la dificultad que aún existe para curar y tratar esta patología. Safi AF et al., 2018 (25) afirman que cuando es diagnosticada la recurrencia locorregional, disminuye significativamente la tasa de supervivencia; sin embargo, el pronóstico y la toma de decisiones terapéuticas dependen en gran medida de la ubicación del tumor $(26,27)$.

En la muestra estudiada, $58 \%$ de los pacientes fueron a óbito, lo que representa un porcentaje alarmante de muertes causadas por esta patología. Debido a que la muestra fue constituida solo por tumores con presencia de invasión ósea, los datos epidemiológicos de este fenómeno parecen reflejar los de la patología en general. En un estudio realizado por Nishi $\mathrm{H}$ et al en 2018 (28), donde se evaluaron 45 casos de CEC de trígono retromolar, la tasa de supervivencia fue de tres años en los 10 pacientes clasificados como T4, lo que está de acuerdo con nuestro estudio, demostrando la severidad de la presencia de invasión.

Finalmente fue posible concluir que, el patrón de invasión ósea infiltrativo de los CEC de la cavidad oral, tuvo asociación estadística con una tasa de supervivencia promedio más baja, siendo así, el análisis de los patrones histopatológicos del CEC con presencia de invasión ósea, ayuda a comprender mejor la patogénesis de este tumor $\mathrm{y}$, consecuentemente, puede usarse como un factor pronóstico para ayudar en las decisiones de tratamiento oncológico.

El desarrollo de métodos diagnósticos basados en las características histopatológicas de la invasión ósea de los CEC, puede ser útil en la elaboración de nuevas estrategias terapéuticas. 


\section{Declaraciones}

Este manuscrito no se ha considerado para publicación en otro lugar y todos los autores han visto y aprobado la versión final.

Los autores declaran no tener ningún conflicto de intereses.

\section{Financiación}

Este manuscrito fue apoyado por el Programa Post-Doctoral de CAPES (Agencia Federal Brasileña de Apoyo y Evaluación de la Educación de Postgrado en el Ministerio de Educación de Brasil).

\section{Correo electrónico:}

Ana Maria Hoyos Cadavid:

ana_hoyosc@hotmail.com

\section{REFERENCIAS BIBLIOGRAFIAS}

1. Taghavi N, Yazdi I. Prognostic factors of survival rate in oral squamous cell carcinoma-. Clinical, histologic, genetic and molecular concepts. Arch Iran Med. 2015; 18: 314-319.

2. Scully C, Bagan J. Oral squamous cell carcinoma overview. Oral Oncol. 2009; 45:301-308.

3. Rivera C. Essentials of oral cancer. Int J Clin Exp Pathol. 2015; 9: 11884-11894.

4. Shield KD, Ferlay J, Jemal A, Sankaranaravanan R, Chaturvedi AK, Bray F, Soeriomataram. The global incidence of lip, oral cavity, and pharyngeal cancers by subsite in 2012. CA Cancer J Clin. 2017; 67: 51-64.

5. Epstein JB, Thriat J, Bensadoun RJ, Barasch A, Murphy BA, Kolnick L, Popplewell L, Maghami E. Oral complications of cancer and cancer therapy: from cancer treatment to survivorship. CA Cancer J Clin. 2012; 62: 400-422.

6. Elmusrati AA, Pilborough AE, Khurram SA, Lambert DW. Cancer- associated fibroblast promote bone invasion in oral squamous cell carcinoma. Br J Cancer. 2017; 117:867-875.

7. Noguti J, De Moura CF, De Jesus GP, Da Silva VH; Hossaka TA, Oshima CT, Ribeiro DA. Metastasis from oral cancer: an overview. Cancer Genomics Proteomics. 2012; 9: 329-335.

8. Namin AW, Zitsch RP, Lavfield LJ. Variability in pathologic interpretation of mandibular invasion. Otolaryngol Head Neck Surg. 2019; 160:1034-1041.

9. Ebrahimi A, Murali R, Gao K, Elliott MS, Clark JR. The prognostic and staging implications of bone invasion in oral squamous cell carcinoma. Cancer. 2011; 117: 4460-4467.

10. Ash CS, Nason RW, Abdoh AA, Cohen MA. Prognostic implications of mandibular invasion in oral cancer. Head Neck. 2000; 22:794-798.

11. Fives C, Nae A, Roche P, O Leary G, Fitzgerald B, Feeley L, Sheahan P. Impact of mandibular invasion on prognosis in oral squamous cell carcinoma four centimeters or less in size. Laryngoscopy. 2017; 127: 849-854.

12. Fried D, Mullins B, Weissler M, Shores C, Zanation A, Hackman T, Shockley W, Hayes N, Chera BS. Prognostic significance of bone invasion for oral cavity squamous cell carcinoma considered T1/T2 by American joint committee on cancer size criteria. Head Neck. 2014; 36:776-781.

13. Muller H, Slootweg PJ. Mandibular invasion by oral squamous cell carcinoma. Clinical aspects. J Craniomaxillofac Surg. 1990; 18:80-84.

14. Wong RJ, Keel SB, Glynn RJ, Varvares MA. Histological pattern of mandibular invasion by oral squamous cell carcinoma. Laryngoscopy. 2000; 110:65-72.

15. Chen YL, Kuo SW, Fang KH, Hao SP. Prognostic impact of marginal mandibulectomy in the presence of superficial bone invasion and the nononcology outcome. Head Neck. 2011; 33:708-713.

16. Jemal A, Siegel R, Ward E, Hao Y, Xu J, Thun MJ. Cancer statistics, 2009. CA Cancer J Clin. 2009; 59: 225-249.

17. Hussein AA, Helder MN, De Visscher JG, Leemans CR, Braakhuis BJ, De Vet HCW, Forouzanfar T. Global incidence of oral and oropharynx cancer in patients younger than 45 years versus older patients: A systematic review. Eur J Cancer. 2017; 82: 115-127.

18. Chi AC, Day TA, Neville BW. Oral cavity and oropharyngeal squamous cell carcinoma an update. CA Cancer J Clin. 2015; 65: 401-421.

19. Michalek J, Brychtova S, Pink R, Dvorak Z. Prognostic and predictive marks for peineural and bone invasion of oral squamous cell carcinoma. Biomed Pap Med Fac Univ Palacky Olomouc Czech Repub. 2019; 21:1-7.

20. Tada T, jimi E, Okamoto M, Ozeki S, Okabe K. Oral squamous cell carcinoma cells induce osteoclast differentiation by suppression of osteoprotegerin expression in osteoblasts. Int J Cancer. 2005; 116:253262.

21. Chuang HC, Su CY, Huang HY, Huang CC, Chien CY, Du YY, Chuang JH. Active matrix metalloproteinase-7 is associated with invasion in buccal squamous cell carcinoma. Mod Pathol. 2008; 21:1444-1450.

22. Takayama $Y$, Mori $T$, Nomura $T$, Shibahara $T$, Sakamoto M. Parathyroid-related protein plays a critical role in bone invasion by oral squamous cell carcinoma. Int J Oncol. 2010; 36:1387-1394.

23. Cui N, Nomura T, Takano N, Wang E, Zhang W, Onda T, Shibahara T. Osteoclast -related cytokines from biopsy specimens predict mandibular invasion by oral squamous cell carcinoma. Exp Ther Med. 2010; 1:755-760. 
24. Brown JS, Browne RM. Factors influencing the patterns of invasion of the mandible by oral squamous cell carcinoma. Int J Oral Maxillofac Surg. 1995; 24:417-426.

25. Safi AF, Kauke M, Grandoch A, Nickenig HJ, Zoller J, Kreppel M. The importance of lymph node ratio for patients with mandibular infiltration of oral squamous cell carcinoma. J Craniomaxillofac Surg. 2018; 46: 1007-1012.

26. Patel SG, Amit M, Yen TC, Liao CT, Chaturvedi P, Agarwal JP, et al. Lymph node density in oral cavity cancer: results of the International Consortium for outcomes research. Br J cancer. 2013; 109: 2087-2095.

27. Shah JP, Gil Z. Current concepts in management of oral cancer-surgery. Oral Oncol. 2009; 45:394-401.

28. Nishi H, Shinozaki T, Tomioka T, Maruo T, Hayashi R. Squamous cell carcinoma of the retromolar trigone: treatment outcomes. Auris Nasus Larynx. 2018; 45:337-342.

Recibido: 14-06-19

Aceptado: 25-02-20 